\title{
Transmission Network Expansion Planning Considering Phase-Shifter Transformers
}

\author{
Celso T. Miasaki, ${ }^{1}$ Edgar M. C. Franco, ${ }^{2}$ and Ruben A. Romero ${ }^{3}$ \\ ${ }^{1}$ Mathematics Department, FUNEC, Av. Mangará, 477, Santa Fe do Sul, SP 15755-000, Brazil \\ ${ }^{2}$ Engineering and Applied Sciences Center (CECE), UNIOESTE, Av. Tarquinio Joslin dos Santos 1300, Foz do Iguazu, \\ PR 85870-000, Brazil \\ ${ }^{3}$ Electrical Engineering Department (DEE), UNESP, Av. Brasil 1200, Ilha Solteira, SP 15385-000, Brazil
}

Correspondence should be addressed to Edgar M. C. Franco, emfranco@unioeste.br

Received 3 October 2011; Accepted 16 January 2012

Academic Editor: Massoud Rashidinejad

Copyright (C) 2012 Celso T. Miasaki et al. This is an open access article distributed under the Creative Commons Attribution License, which permits unrestricted use, distribution, and reproduction in any medium, provided the original work is properly cited.

\begin{abstract}
This paper presents a novel mathematical model for the transmission network expansion planning problem. Main idea is to consider phase-shifter (PS) transformers as a new element of the transmission system expansion together with other traditional components such as transmission lines and conventional transformers. In this way, PS are added in order to redistribute active power flows in the system and, consequently, to diminish the total investment costs due to new transmission lines. Proposed mathematical model presents the structure of a mixed-integer nonlinear programming (MINLP) problem and is based on the standard DC model. In this paper, there is also applied a specialized genetic algorithm aimed at optimizing the allocation of candidate components in the network. Results obtained from computational simulations carried out with IEEE-24 bus system show an outstanding performance of the proposed methodology and model, indicating the technical viability of using these nonconventional devices during the planning process.
\end{abstract}

\section{Introduction}

Transmission network planning begins with the establishment of power demand growth scenarios, in accordance with forecasts along the time. Given these scenarios, one can verify the eventual need to broaden and to strengthen the network. In case electric service conditions are not satisfied, there should be proposed a plan that has coherence among the power supply availability, demand, and installation of new equipments in the network. Integration of these new equipments in the network, aimed at maintaining suitable technical and operating conditions, requires planning of the allocation of such reinforcement.

Main objective of the transmission expansion planning is to obtain the optimal expansion plan, while fulfilling operating and economic constraints.

Formulation of a mathematical representation for the transmission expansion planning problem begins with some assumptions, where accuracy and complexity are considered in the model construction. Regularly, the problem is represented by a Mixed-Integer Nonlinear Programming (MINLP) problem that presents many local optima solutions for real-life systems. This high number is due to the possible expansion plans that shows the association of the specified optimal operational mode. Therefore, a basic problem consists in defining the least-cost expansion alternative that satisfies all operating constraints.

In static long-term transmission expansion planning (typically with a planning horizon of more than 5 years), all investments are carried out in a single-year planning horizon, whereas for the multistage it is divided into several stages.

Static planning is aimed at searching where and what type of circuits should be constructed for the network to operate properly. This type of planning applies some simplifications; for instance, voltage magnitudes constraints are neglected, albeit in short-term planning are essential. Voltage and stability problems are not taken into account in this 
approach either. Even though considering these simplifications, the problem still remains complex with unknown optima solutions for various real-life systems.

In the technical literature, DC and transportation models are static mathematical models often used to solve the transmission expansion planning problem. These models consider only the addition of transmission lines (TLs) and conventional transformers.

Here a novel transmission expansion strategy is proposed. An improved model considers the inclusion of a new kind of device, in this case, a flexible alternating current transmission system (FACTS) device.

The literature concerning the use of FACTS devices is wide. However, most papers treat only the operational improvement by using FACTS devices [1-3]. This topic is considered as the operation planning, in which FACTS devices are used, for example, to redistribute the active power flow in order to eliminate congestion problems and/or to optimize the power dispatch. In this way, the PS is considered a FACTS device with the characteristic of redistributing the active power flow.

Feasibility of employing the PS as a candidate component in the long-term transmission expansion planning process is analyzed regarding a static and centralized planning model. Nevertheless, the present model can be extended to the multistage planning [4], to the competitive planning [5], or to the planning with security constraints [6].

Instead of trying to consider all functions concerning the planning problem in a single model, this work is focused solely on the core of the network synthesis; for which the mathematical modeling and the solution technique is addressed.

The proposed model is based on the DC model, which is the most employed one in planning problems; consequently, only the active power flow is considered. Other aspects such as performance analysis (reliability and stability analysis, reactive planning, AC power flow, and short-circuit calculation) relevant to transmission expansion are beyond the scope of this paper. Nonetheless, in general, after obtaining a basic solution, all those analyses can be carried out.

\section{Mathematical Model}

This section introduces the classical mathematical model and the proposed one.

2.1. Classical Model. The mathematical formulation of the DC model for transmission network expansion planning problem, when considering solely the installation of transmission lines and/or conventional transformers, assumes the following form:

$$
\text { Minimize } \quad v=\sum_{(i, j)} c_{i j} n_{i j}
$$

subject to

$$
\begin{gathered}
S \cdot f+g=d, \\
f_{i j}-\gamma_{i j} \cdot\left(n_{i j}^{0}+n_{i j}\right) \cdot\left(\theta_{i}-\theta_{j}\right)=0,
\end{gathered}
$$

$$
\begin{gathered}
\left|f_{i j}\right| \leq\left(n_{i j}^{0}+n_{i j}\right) \cdot \bar{f}_{i j}, \\
0 \leq g_{i} \leq \overline{g_{i}}, \\
0 \leq n_{i j} \leq \overline{n_{i j}}, \\
n_{i j} \quad \text { Integer, } \\
f_{i j} \quad \text { Unbounded, } \\
\theta_{j} \quad \text { Unbounded, } \\
(i, j) \in \Omega .
\end{gathered}
$$

The objective function (1) represents the investment cost due to new transmission lines during the specified planning horizon.

The first set of constraints (2) represents Kirchhoff's Current Law (KCL) equations (one constraint per bus), and the second one (3) represents Kirchhoff's Voltage Law (KVL) (one constraint per branch). In this model, the transmission lines or regular transformers are represented without distinction by decision variables $n_{i j}$.

Set of constraints (4) refers to the capacity of transmission circuits (lines and/or transformers) in terms of the required absolute value since the power flow can flow in both ways.

Constraint (5) represents the limits for generation buses and (6) represents the limits for the added circuits at each candidate path $i$ - $j$. Remaining constraints refer to the selfcharacteristics of the variables.

From the operational research standpoint, system (1)(10) is a MINLP whose resolution is complex, especially for large-size electric systems. Main source of complexity in the problem is due to variables $n_{i j}$ that need to be integer.

For some types of algorithms utilized for the transmission network planning problem, it is more suitable to carry out alterations to the basic modeling for allowing the application of the solution techniques. An alteration commonly used is the insertion of new variables that represent the load shedding associated with all load buses of the system. This resource can also be seen as an artificial generation aimed at turning the problem always viable during computational implementations [7]. Note that this artificial generation, from the mathematical standpoint, is solely an increase in the number of variables of the original problem. Additionally, all variables corresponding to the artificial generators in the final solution should be equal to zero, so both problems (original and modified) will have equivalent solutions.

2.2. Model with Phase Shifters. When PS is considered in the transmission network planning problem, the DC model assumes the following form:

$$
\text { Minimize } \quad v=\sum_{(i, j)} c_{i j} n_{i j}+\sum d_{i j} \cdot\left(n_{i j}^{0}+n_{i j}\right) \cdot \delta_{i j}+\alpha \sum r_{k}
$$


subject to

$$
\begin{gathered}
S \cdot f+g+r=d, \\
f_{i j}-\gamma_{i j} \cdot\left(n_{i j}^{0}+n_{i j}\right) \cdot\left(\theta_{i}-\theta_{j}+\varphi_{i j} \cdot \delta_{i j}\right)=0, \\
\left|f_{i j}\right| \leq\left(n_{i j}^{0}+n_{i j}\right) \cdot \bar{f}_{i j}, \\
0 \leq g_{i} \leq \overline{g_{i}}, \\
0 \leq n_{i j} \leq \overline{n_{i j}}, \\
0 \leq r_{k j} \leq d_{k}, \\
n_{i j} \quad \text { Integer, } \\
f_{i j} \quad \text { Unbounded, } \\
\theta_{j} \quad \text { Unbounded, } \\
\varphi_{i j} \quad \text { Unbounded, } \\
\delta_{i j} \quad \text { Unbounded, } \\
(i, j) \in \Omega .
\end{gathered}
$$

The objective function (11) corresponds to the investment cost. First two terms refer to the costs due to the insertion of transmission lines and PS, respectively. Last term refers to a penalty for the configuration under analysis, due to the presence of artificial generation. The penalty parameter $\alpha$ should be properly weighted in order to turn those configurations less attractive with artificial generation.

In constraint (12), corresponding to KCL, vectors $g$ and $r$ indicate existent and artificial generations, respectively.

Installation of PS in one or more lines can be represented as the combination of the buses' angles and the angle supplied by the equipment.

Angle between terminal voltages of a transmission line can be modified by installing a PS. Therefore, power flow equations (KVL) are affected when these devices are inserted. Thus, the function of PS appears in the KVL, which redirects the active power flow.

The PS is considered a component with negligible reactance that can be placed in series with a transmission line or a conventional transformer.

In this work, the angular difference of the PS was considered an unbounded variable; however, a limit can be set without modifying the mathematical model significantly.

Presence of variable $\delta_{i j} \in\{0,1\}$ in (13) makes this relation even more nonlinear in relation to the DC model and,

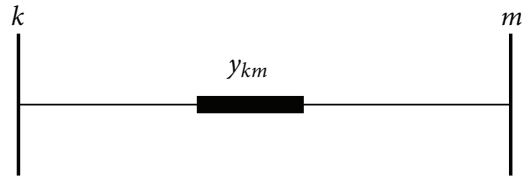

Figure 1: Transmission line.

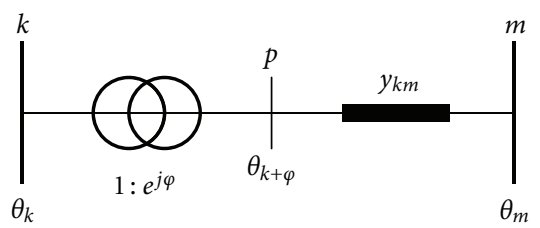

Figure 2: Transmission line and phase-shifter transformer.

TABLE 1: Lines data of the 3 buses system.

\begin{tabular}{lcc}
\hline Line & Reactance $(\mathrm{pu})$ & Maximum flow $(\mathrm{MW})$ \\
\hline $1-2$ & 0.333 & 35 \\
$1-3$ & 0.500 & 40 \\
$2-3$ & 0.500 & 40 \\
\hline
\end{tabular}

consequently, more complex since four types of variables $\left(n_{i j}, \theta_{j}, \varphi_{i j}\right.$, and $\left.\delta_{i j}\right)$ are multiplied.

Set of constraints (14), (15), (16), and (17) refers to the transmission capacity of the circuits, limits of generation buses, limits of circuits added in each candidate path $i-j$, and limits of artificial generation buses, respectively. Remaining constraints refer to the characteristics of variables.

Therefore, the proposed mathematical model is more complex than the classical one, due to the characteristics of a PS.

In traditional models, a transmission line or a conventional transformer in a path $k-m$ can be represented in Figure 1.

The PS is considered with zero impedance connected in series with a transmission line or a conventional transformer in path $k-m$ as shown in Figure 2.

It is also considered that when a path $k-m$ is selected, the PS is allocated to every transmission line present in path $k-m$. Therefore, the number of PS at each selected path is equal to $n \mathrm{PS}_{i j}=\left(n_{i j}^{0}+n_{i j}\right) \cdot \delta_{i j}$. The PS can be allocated in existing transmission lines as well as in those created during the optimization process.

Problem (11)-(23) represents a MINLP, which is more complex than the model (1)-(10). However, metaheuristic techniques employed for $(1)-(10)$ can also be applied to (11)-(23) after performing some modifications.

2.3. Illustrative Example: 3-Bus System. An example consisting of a network with three buses is presented in order to illustrate the application of the PS. System data is shown in Tables 1 and 2 .

Results obtained by performing the linearized DC power flow are illustrated in Figure 3. 
TABLE 2: Generation and demand data of the 3-buse system.

\begin{tabular}{lcc}
\hline Bus & Generation (MW) & Demand (MW) \\
\hline 1 & 70 & 0 \\
2 & 0 & 60 \\
3 & 0 & 10 \\
\hline
\end{tabular}

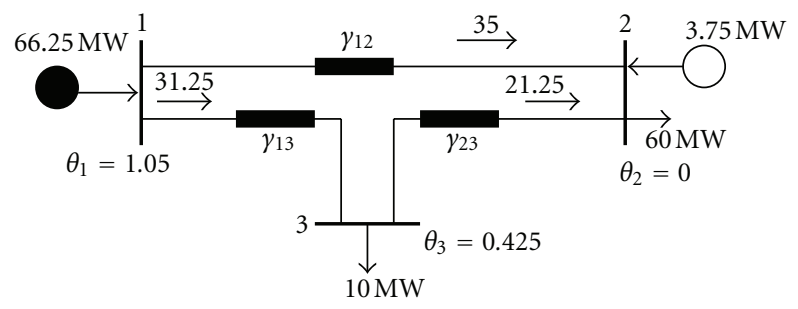

Figure 3: Three-bus system.

Notice that there is a load shedding of $r_{2}=3.75 \mathrm{MW}$ at bus 2 . Therefore the system is not operating adequately and new transmission lines are required. On the other hand, lines $1-3$ and 2-3 are operating below their capacity. In this case, there is no possibility of increasing the transmitted power through the lines without overloading line 1-2. An interesting solution for this problem is to install a PS at 1-3 (see Figure 4), so the system operates without load shedding.

Thus, this example shows how a PS is able to redirect the active power flow. This property will be employed in the long-term transmission planning, in which basic components are transmission lines, conventional transformers, and PS.

2.4. Phase-Shifter Transformer Features. PSs have the ability to redirect active power flows in the network. This feature provides a dynamic operational mode since it makes increasing the utilization of existing circuits possible. Consequently, as it can be verified from relation (13), the PS acts directly on KVL.

Another important aspect is the use of relaxed models. In general, optimal solutions for relaxed models are not feasible for more accurate or constrained ones. Thus, it is probable that the optimal solution obtained by the transportation model, where the KVL (13) is relaxed (dropped), presents the KVL constraints violated in the DC model. With the inclusion of PS in determined positions, a feasible solution obtained with transportation model becomes also feasible for the DC model since the KCL constraints are already satisfied for both models. However, an efficient inclusion should be carried out in order to satisfy the optimality conditions of the DC model, once there exist expansion proposals with lower costs. It is worthwhile to notice that if PS costs are equal to zero, then the optimal solution for the transportation model and the DC model presents the same added lines, that is, presents the same topology for both mathematical models. Only difference with the conventional approach is that some PS are included in order to satisfy the KVL.

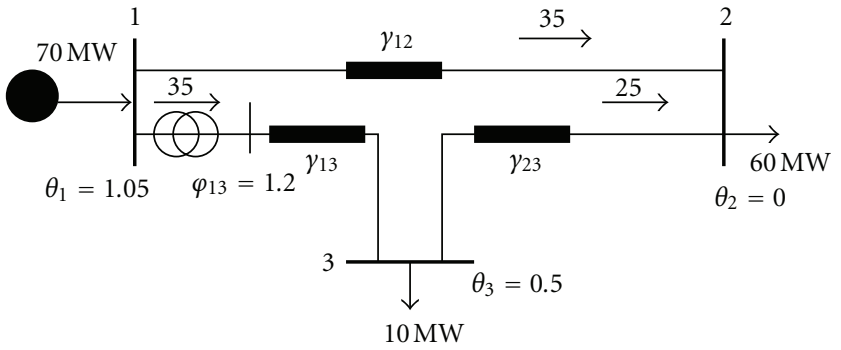

FIGURE 4: Load flow solution with a phase shifter.

The primary objective of this work is to verify the operation of PS and the technical feasibility of considering such type of equipment in long-term transmission expansion planning. In case a reduced-cost PS is employed, the optimal solution will be the same of the transportation model with addition of PS. On the other hand, higher costs will inhibit the presence of PS in the optimal solution, tending to the solution given by classical DC model. Finally, if the costs are competitive to transmission lines, an intermediary solution will be provided.

\section{Solution Mathodology}

Metaheuristic algorithms are specially suited for problems that present large search space with many local optima, such as the transmission expansion planning problem. The nonlinearity of the problem concerning the KVL is higher than the conventional model, thus degenerating even more the performance of more accurate methods. For instance, simulated annealing, genetic algorithms, and tabu search represent efficient methods for solving such problems. This work employed a modified version of the genetic algorithm presented in $[4,8]$ for solving the mathematical optimization problem. Indeed, extra modifications become necessary in order to guarantee the acceptable performance of the genetic algorithm.

\section{Genetic Algorithm}

This section presents the genetic algorithm developed for the planning problem considering the addition of PS.

4.1. Encoding. Each individual in a population (chromosome) is a proposed solution for the problem. In this work, an individual is encoded considering only the integer and binary variables. Remaining variables (continuous variables) are obtained from the linear programming (LP) solution. Thus, transmission lines and transformers are represented by decimal encoding (variable $n_{i j}$ ), whereas the PSs are represented by binary variables $\left(\delta_{i j}\right)$.

An example of this chromosome of length $2 n l$ is shown in Figure 5. The first $n l$ positions show the number of transmission lines added for each configuration and the last $n l$ positions indicate whether a PS was added or not in each path. The existing transmission lines are not coded but they 


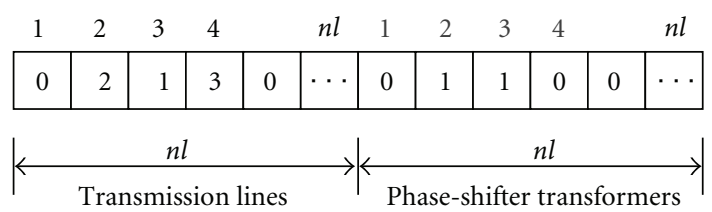

FIgURE 5: Encoding proposal (chromosome).

are taken into account when operational variables are calculated, as well as when PSs are added in series to the transmission lines at each path. It can be noticed that in path 2, two transmission lines have been added with the corresponding PS. In path 3, one transmission line and the necessary number of PSs have been added, and so on.

In [8], an excellent performance of the genetic algorithm was obtained with the coding above, which is also in accordance with the coding proposals suggested for genetic algorithms presented in $[4,9,10]$.

4.2. Objective Function and Infeasibility. The objective function of any solution proposal is found by solving an LP problem. The LP determines the exact values of the operational variables, which makes verifying the operation feasibility of a determined investment proposal possible, that is, whether the system presents load shedding to the implemented expansion proposal. Considering that an investment proposal $s$ is characterized by variables $n_{i j}^{s}$ and $\delta_{i j}^{s}$, the load shedding is obtained by solving the following LP problem:

$$
\text { Minimize } w^{s}=\sum_{k \in \Gamma} r_{k}
$$

subject to

$$
\begin{gathered}
S \cdot f+g+r=d, \\
f_{i j}-\gamma_{i j} \cdot\left(n_{i j}^{0}+n_{i j}^{s}\right) \cdot\left(\theta_{i}-\theta_{j}+\varphi_{i j} \cdot \delta_{i j}^{s}\right)=0, \\
\left|f_{i j}\right| \leq\left(n_{i j}^{0}+n_{i j}^{s}\right) \cdot \bar{f}_{i j}, \\
0 \leq g_{i} \leq \bar{g}_{i}, \\
0 \leq r_{k} \leq d_{k},
\end{gathered}
$$

$f_{i j}$ Unbounded,

$\theta_{j}$ Unbounded,

\begin{tabular}{|c|c|c|c|c|c|c|c|c|c|c|c|}
\hline \multicolumn{12}{|c|}{$\begin{array}{l}\text { Crossover } \\
\text { point }\end{array}$} \\
\hline 1 & 2 & 3 & 4 & & $n l$ & 1 & 2 & 3 & 4 & & $n l$ \\
\hline 0 & 2 & 1 & 3 & 0 & $\cdots$ & 0 & 1 & 1 & 0 & 0 & $\cdots$ \\
\hline & & & & & & & & & & & \\
\hline 1 & 0 & 1 & 0 & 2 & $\cdots$ & 1 & 0 & 0 & 0 & 0 & $\cdots$ \\
\hline
\end{tabular}

$\varphi_{i j}$ Unbounded,

\begin{tabular}{|c|c|c|c|c|c|c|c|c|c|c|c|}
\hline en & en & 1 & 0 & 0 & $\cdots$ & 0 & 12 & 1 & 0 & 0 & $\cdots$ \\
\hline 1 & 0 & 眮 & 阳 & 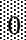 & $\cdots$ & 1 & 0 & 睢苗 & 閶 & (1) & \\
\hline
\end{tabular}

$$
(i, j) \in \Omega
$$

(a) Before crossover

(b) After crossover

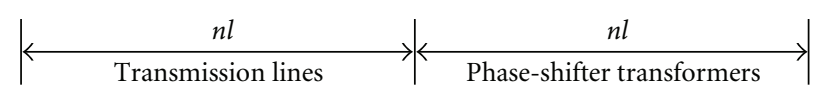

FIGURE 6: Single point crossover.

For each solution proposal, the objective function is calculated with the following expression:

$$
v^{s}=\sum_{(i, j) \in \Omega} c_{i j} n_{i j}^{s}+\sum d_{i j} \cdot\left(n_{i j}^{0}+n_{i j}^{s}\right) \cdot \delta_{i j}^{s}+\alpha \cdot w^{s} .
$$

In the genetic algorithm, every solution proposal is considered, including the infeasible ones. The infeasible configurations (with load shedding) are eliminated gradually by selection process, since these configurations are penalized by parameter $\alpha$ in the objective function.

4.3. Selection Process. The selection is based on tournament with $k=2$, that is, a game where two topologies of the current population participate. Recent publications show that selection by tournament is the most efficient method as long as parameter $k$ is set adequately.

4.4. Crossover. The single point crossover was employed in this work. The crossover point is chosen randomly and a descendant, which has a parcel of its parents from the crossover point, is created. The random point was generated from an interval of 1 to $(n l-1)$, that is, considering only the first half of the chromosome. In the second part the same operation is executed as shown in Figure 6. The objective of the operation is to transmit the existing information, which is present in both sectors of the chromosome to only one descendant in order to avoid quality degeneration.

4.5. Mutation. The mutation operator acts in the following form. Considering transmission lines, the application of mutation operation means the addition or removal of one transmission line added during the optimization process $\left(n_{i j}+1\right.$ or $\left.n_{i j}-1\right)$. For PS, it means the change of an allele, that is, inclusion or exclusion of the PS in the path. 


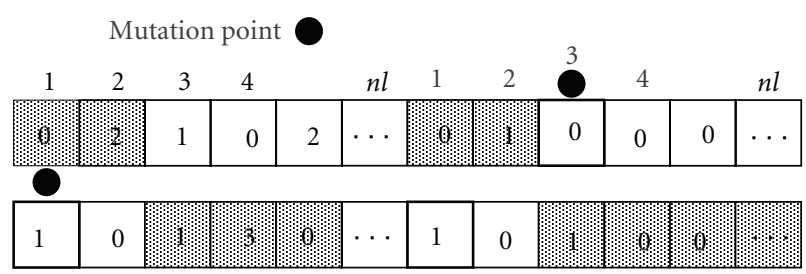

(a) Before mutation

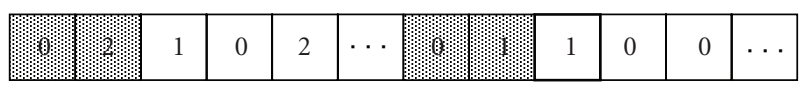

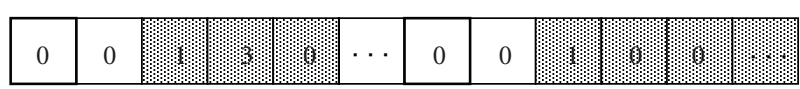

(b) After mutation

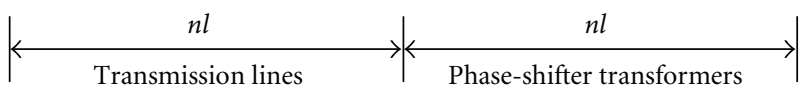

Figure 7: Mutation.

The mutation operation should be executed respecting the following conditions:

(1) the maximum number of transmission lines in the path;

(2) before adding a PS in the selected path, the existence of a transmission line must be checked; if it is an empty path, a transmission line has also to be added;

(3) when a transmission line is removed, the corresponding PS is removed, in case it exists.

Concluding, a PS can only be inserted to an existing transmission line, whereas the number of PS is equal to the number of transmission lines in a branch. In Figure 7, two cases of mutation are presented. The first case shows the random addition of PS to an existing transmission line, and the second case, mutation is applied to the transmission line that is removed (in this case there is no transmission line in the base topology); then the PS is removed in order to keep the process coherent.

4.6. Proposed Genetic Algorithm. The general structure of the implemented genetic algorithm is similar to that presented in [8], except for some modifications. The basic structure of the algorithm is the following.

(1) Set the control parameters and generate the initial population. Make the initial population the current population.

(2) Calculate the objective function of the current population by solving one LP for each element (topology) of the current population. Update the incumbent solution whenever possible.

(3) If the stopping criterion is satisfied, stop the process. Otherwise go to step 4.

(4) Execute selection by tournament with $k=\{2 ; 3\}$.

(5) Execute one point crossover.
(6) Implement specialized mutation.

(7) Form the current population and go to step 2.

Some details of the algorithm are presented in the next section.

4.7. Details and Improvement of the Genetic Algorithm. We present, briefly, details of the algorithm and some improvements made to the genetic algorithm.

4.7.1. Generation of the Initial Population. Generation of the initial population is made by a controlled random process. Basically, it defines the number of paths in which the transmission lines are added and the maximum number of PS. Regarding transmission lines are defined randomly number of branches where the lines are inserted, position, and number of transmission lines (subject to the limits of added lines). In the case of PS, the number of branches and the position are selected randomly. In general, experience in transmission planning indicates that the number of branches to be added should be small, whereas the number for PS should be even smaller.

The performance coefficient (35) can be employed for ranking the most interesting transmission lines. Observe that lines with a larger capacity that is also cost-effective have the priority in the addition:

$$
\mathrm{PC}_{i j}=\frac{\overline{f_{i j}} \gamma_{i j}}{c_{i j}} .
$$

After ranked, the circuits are separated into two groups with different size $(75 \%, 25 \%)$, whereas the largest one presents the most interesting transmission lines in terms of capacity usage. The initial population is formed by $80 \%$ up to $100 \%$ of elements belonging to the largest group. Another option to the initial population generation is to employ constructive heuristic algorithms as in $[11,12]$.

4.7.2. Control Parameters: Crossover and Mutation Rates. The employed crossover rate was $R_{C}=0.8$. Two mutation rates were employed: $R_{M}=0.1$ or $R_{M}=0.6$ (the value depends on the current population diversification level). The mutation rate is defined differently from the classical concept and it applies for an entire solution proposal (topology). For example, a rate of 0.1 means that there is $10 \%$ of probability for a topology to suffer mutation. The population size varied from 40 to 200 elements.

4.7.3. Mutation Details. Mutation is executed in the following way: the power flow of each topology is stored in four matrices considering the load level of each circuit. They are separated in intervals of $25,50,75$, and $100 \%$ of capacity. The load level is calculated by means of the relationship (36):

$$
\mathrm{FC}_{i j}=\frac{f_{i j}}{\left(n_{i j}^{0}+n_{i j}\right) \cdot \overline{f_{i j}}} .
$$

Mutation operation is executed based on the probability of $70 \%$ of circuit removal and $30 \%$ of circuit addition. In case 
TABLE 3: Five transmission expansion plans considering low-cost phase-shifter transformers.

\begin{tabular}{|c|c|c|c|c|c|c|c|c|c|c|}
\hline \multirow{2}{*}{ Circuits } & \multicolumn{2}{|c|}{ Plan $P_{0}$} & \multicolumn{2}{|c|}{ Plan $P_{1}$} & \multicolumn{2}{|c|}{ Plan $P_{2}$} & \multicolumn{2}{|c|}{ Plan $P_{3}$} & \multicolumn{2}{|c|}{ Plan $P_{4}$} \\
\hline & $\mathrm{TL}$ & PS & $\mathrm{TL}$ & PS & $\mathrm{TL}$ & PS & $\mathrm{TL}$ & PS & $\mathrm{TL}$ & PS \\
\hline $\mathbf{n}_{01-03}$ & & & & & & & & 1 & & \\
\hline $\mathbf{n}_{03-09}$ & & & & & & 1 & & & & \\
\hline $\mathbf{n}_{04-09}$ & & & & & & 1 & & & & \\
\hline $\mathbf{n}_{03-24}$ & & & & & 1 & & & & & \\
\hline $\mathbf{n}_{06-10}$ & 1 & & 1 & & 1 & & 1 & & 1 & \\
\hline $\mathbf{n}_{07-08}$ & 2 & & 2 & & 1 & & 2 & & 2 & \\
\hline $\mathbf{n}_{08-09}$ & & 1 & & 1 & & & & & & \\
\hline $\mathbf{n}_{08-10}$ & & & & & & & & & 1 & \\
\hline $\mathbf{n}_{10-12}$ & & & & & 1 & & & & & 1 \\
\hline $\mathbf{n}_{11-14}$ & & 1 & & 1 & & & & & & \\
\hline $\mathbf{n}_{12-23}$ & & & & 1 & & & & 1 & & \\
\hline $\mathbf{n}_{13-23}$ & & & & & & & & & & 1 \\
\hline $\mathbf{n}_{14-16}$ & 1 & & 1 & & 1 & & & & 1 & \\
\hline $\mathbf{n}_{15-16}$ & & & & & & & & 1 & & \\
\hline $\mathbf{n}_{16-17}$ & & & 2 & & 2 & & & 1 & 1 & \\
\hline $\mathbf{n}_{16-19}$ & & & 1 & & 1 & & & & & \\
\hline $\mathbf{n}_{17-18}$ & & & 1 & & 1 & & & & & \\
\hline $\mathbf{n}_{17-22}$ & & & & & & & & 1 & & \\
\hline $\mathbf{n}_{19-22}$ & & & & & & 2 & & & & \\
\hline $\mathbf{n}_{13-14}$ & & & & & & & 1 & 1 & & \\
\hline Partial cost & 102 & 4 & 226 & 6 & 310 & 8 & 110 & 14 & 188 & 4 \\
\hline Total cost & & & & & & & & & & \\
\hline
\end{tabular}

that circuit addition operation is selected, a random selection is carried out over the branches from the matrix with most loaded branches (up to 75\%). If the matrix is empty, the next load level matrix is searched. For the circuit removal operation, the search starts from the least loaded branches (matrices with branches on $25 \%$ and $50 \%$ of its capacity). With this strategy, transmission lines with low utilization are removed from the system, whereas in regions where higher utilization is observed lines are inserted.

4.7.4. Stopping Criterion. The algorithm stops when a defined total number of iterations is reached or when there is no improvement of the incumbent solution after a specified number of iterations.

4.7.5. Elitism and Diversification Control. The population diversity is controlled by changing the mutation rate. A measurement of diversity is given by (37):

$$
R_{\text {Div }}=\frac{\text { Pop }-\mathrm{NR}}{\text { Pop }} \text {. }
$$

The diversification rate is calculated after mutation. If this rate is below $50 \%$, there is used a mutation rate of 0.6 ; otherwise a rate of 0.1 is considered. This mechanism was applied aimed at maintaining diversity and at exploring new search spaces.
Another strategy used was the elitism, in which the parent topologies are compared with the descendants and then the two best topologies are preserved in the current population.

\section{Tests and Results}

In order to analyze the performance of the proposed genetic algorithm and to demonstrate viability of the mathematical model with PS, tests were carried out with the IEEE-24 bus system. This system has 41 circuit paths, $8550 \mathrm{MW}$ of load, generation capacity of $10215 \mathrm{MW}$, and five different generation plans whose data is present in [5].

For all simulations, a fixed cost for the PS was adopted, that varies among 2 and 120 million of dollars. In all the tests was used a value of $\alpha=10^{6} \mathrm{US} \$ / \mathrm{MW}$.

The simulations were divided in three stages. In the first stage, equipments of low value were added. In the following stage, equipments of high value were added. Finally, in the last stages, intermediate costs were adopted; that is, in each stage different values were fixed for the PS.

5.1. Low-Cost Phase Shifters. With the purpose of testing the algorithm, the first simulations set the cost of PS as low as $d_{i j}=2 \times 10^{6}$ (US\$). Table 3 shows the results obtained. 
TABLE 4: Five transmission expansion plans considering high-cost phase-shifter transformers.

\begin{tabular}{|c|c|c|c|c|c|c|c|c|c|c|}
\hline \multirow{2}{*}{ Circuits } & \multicolumn{2}{|c|}{ Plan $P_{0}$} & \multicolumn{2}{|c|}{ Plan $P_{1}$} & \multicolumn{2}{|c|}{ Plan $P_{2}$} & \multicolumn{2}{|c|}{ Plan $P_{3}$} & \multicolumn{2}{|c|}{ Plan $P_{4}$} \\
\hline & $\mathrm{TL}$ & PS & $\mathrm{TL}$ & PS & $\mathrm{TL}$ & PS & $\mathrm{TL}$ & PS & $\mathrm{TL}$ & PS \\
\hline $\mathbf{n}_{01-05}$ & & & 1 & & 1 & & & & & \\
\hline $\mathbf{n}_{03-24}$ & & & 1 & & 1 & & & & 1 & \\
\hline $\mathbf{n}_{06-10}$ & 1 & & 1 & & 1 & & 1 & & 1 & \\
\hline $\mathbf{n}_{07-08}$ & 2 & & 2 & & 1 & & 2 & & 2 & \\
\hline $\mathbf{n}_{09-11}$ & & & & & & & & & 1 & \\
\hline $\mathbf{n}_{10-12}$ & 1 & & & & 1 & & 1 & & 1 & \\
\hline $\mathbf{n}_{11-14}$ & & & & & & & & & 1 & \\
\hline $\mathbf{n}_{14-16}$ & 1 & & 1 & & 1 & & 1 & & 1 & \\
\hline $\mathbf{n}_{15-24}$ & & & 1 & & 1 & & & & & \\
\hline $\mathbf{n}_{16-17}$ & & & 2 & & 2 & & 1 & & 1 & \\
\hline $\mathbf{n}_{16-19}$ & & & 1 & & & & & & & \\
\hline $\mathbf{n}_{17-18}$ & & & 2 & & 2 & & & & & \\
\hline $\mathbf{n}_{20-23}$ & & & & & & & 1 & & & \\
\hline Partial cost & 152 & 0 & 390 & 0 & 392 & 0 & 218 & 0 & 346 & 0 \\
\hline Total cost & & & & & & & & & & \\
\hline
\end{tabular}

TABLE 5: Plan $P_{1}$ of the system IEEE-24bus considering intermediary-cost phase-shifter transformers.

\begin{tabular}{|c|c|c|c|c|c|c|}
\hline \multirow{2}{*}{ Circuits } & \multicolumn{2}{|c|}{ Plan $P_{0}$} & \multicolumn{2}{|c|}{ Plan $P_{1}$} & \multicolumn{2}{|c|}{ Plan $P_{2}$} \\
\hline & $\mathrm{TL}$ & PS & $\mathrm{TL}$ & PS & $\mathrm{TL}$ & PS \\
\hline $\mathbf{n}_{03-24}$ & & 1 & & 1 & & 1 \\
\hline $\mathbf{n}_{06-10}$ & 1 & & 1 & & 1 & \\
\hline $\mathbf{n}_{07-08}$ & 2 & & 2 & & 2 & \\
\hline $\mathbf{n}_{08-10}$ & & 1 & & & & \\
\hline $\mathbf{n}_{09-12}$ & & & & 1 & & \\
\hline $\mathbf{n}_{10-11}$ & 1 & & 1 & & & \\
\hline $\mathbf{n}_{10-12}$ & & & & & 1 & \\
\hline $\mathbf{n}_{12-23}$ & & & & & & 1 \\
\hline $\mathbf{n}_{14-16}$ & 1 & & 1 & & 1 & \\
\hline $\mathbf{n}_{16-17}$ & 2 & & 2 & & 2 & \\
\hline $\mathbf{n}_{16-19}$ & 1 & & 1 & & 1 & \\
\hline $\mathbf{n}_{17-18}$ & 1 & & 1 & & 1 & \\
\hline Partial cost & 276 & 110 & 276 & 110 & 276 & 110 \\
\hline Total cost & & & & & & \\
\hline
\end{tabular}

A very interesting fact in the simulations with low-cost PS refers to transmission lines. For the five plans illustrated in Table 3, it is noticed that the lines added by the proposed specialized genetic algorithm were the same of the optimal solution for the transportation model. This fact was confirmed by utilizing a branch-and-bound algorithm presented in [13].

Results also confirmed that the optimal solution for the transportation model is not feasible for the DC model since KVL constraints are violated. Nonetheless, during the simulation, this problem was overcome by adding low-cost PS in strategic branches.
5.2. High-Cost Phase Shifters. Here there is considered $d_{i j}=$ $120 \times 10^{6}$ (US\$) for high-cost PS. For the same five generation plans, obtained results are illustrated in Table 4.

Again, allocation of lines deserves importance. Simulations carried out with high-cost PS indicate that there was no addition of PS in none of the five plans of expansion of the system. The justification for the absence of PS, in all the topologies, is that they are too expensive. Thus, they did not take part of the optimal solutions. Consequently, there is a tendency of solely adding transmission lines.

Another interesting fact that deserves emphasis is that the results are the same found to the conventional DC model. 
5.3. Intermediary-Cost Phase Shifters. PSs with arbitrary intermediary costs were employed in order to produce expansion proposals with intermediary values when compared to extreme solutions obtained in previous simulations. The PS was considered with cost of $d_{i j}=55 \times 10^{6}$ (US\$) for every branch in plan $P_{1}$. Obtained results are shown in Table 5.

The proposed algorithm found alternative optimal solutions for the plan $P_{1}$. Observe that circuit 10-11, which is not present in the previous cases (PS with low and high costs), appears now. Notice that in the three topologies found for the plan $P_{1}$ were added two phase-shifter transformers in the network.

It is important to mention that all tests have been carried out for PS with nonrealistic cost values in order to test, from the theoretical point of view, the feasibility of modeling such devices as expansion components for electrical systems. Additional network transmission planning bibliography can be found in [14]. It should be observed that this novel model could be solved by other metaheuristics as presented in $[9,15,16]$ after small adaptations.

\section{Conclusions}

Nowadays, modern elements, such as FACTS devices, are playing an important role in transmission systems. In this way, inclusion of such devices jointly with classical components is of importance for the transmission expansion planning problem. Thus, this work was aimed at presenting the technical feasibility of considering phase-shifter transformers as components for the long-term transmission expansion planning, jointly with conventional transformers and transmission lines.

A novel methodology was proposed for the inclusion of phase-shifter transformers in the mathematical model that represents the transmission planning problem. The proposed model is more complex than the model DC. However, the mathematical problem was solved adequately with genetic algorithms.

Tests have shown the model consistency as well as the high performance of the algorithm.

This contribution extends the utilization of classical components during the expansion-planning problem, to modern elements, such as the FACTS devices.

\section{Nomenclature}

v: Investment costs (US\$)

$c_{i j}$ : Cost of a circuit that can be added in path $i-j$ (US\$)

$n_{i j}$ : Number of circuits added in path $i-j$

S: Node-branch transposed incidence matrix of the system

$f$ : Active power flow composed by elements $f_{i j}$

$g$ : Generation composed by elements $g_{k}$

$d$ : Demand of the buses

$f_{i j}$ : Total active power flow through path $i-j$

$\gamma_{i j}$ : Susceptance of one circuit in path $i-j$

$n_{i j}^{o}$ : Base case total number of circuits $\theta_{i}: \quad$ Phase angle of bus $i$

$\overline{f_{i j}}$ : Active power flow limit of one circuit in path

$i-j$

$g_{i}: \quad$ Generation level of bus $i$

$\overline{g_{i}}: \quad$ Generation capacity limit of bus $i$

$\overline{n_{i j}}$ : Number of circuits that can be added in path $i-j$

$\Omega: \quad$ Set of all paths

$d_{i j}$ : $\quad$ Fixed cost of a PS in path $i-j$

$\delta_{i j}$ : $\quad$ Represents the presence (1) or not (0) of a PS in path $i-j$

$\alpha$ : $\quad$ Penalty factor due to load shedding

$r_{k}$ : Artificial generator at load bus $k$

$\Gamma: \quad$ Set of buses with load

$\varphi_{i j}: \quad$ Angular difference of a PS in path $i-j$

$n \mathrm{PS}_{i j}$ : Number of PS added in path $i-j$

$n l$ : Total number of paths in the network

$n_{i j}^{s}$ : Number of the circuits added in path $i-j$, considering an investment proposal $s$ given by the specialized genetic algorithm

$\delta_{i j}^{s}$ : $\quad$ Represents the presence (1) or not (0) of a PS in path $i$ - $j$, considering an investment proposal $s$ given by the specialized genetic algorithm

$w^{s}$ : Load shedding costs of a configuration (US\$)

$\mathrm{PC}_{i j}$ : Performance coefficient of one transmission line in path $i-j$

$R_{C}: \quad$ Crossover rate

$R_{M}$ : Mutation rate

$\mathrm{FC}_{i j}$ : Flow utilization coefficient of one transmission line in path $i-j$

$R_{\text {Div }}:$ Population diversification ratio (\%)

NR: Total number of repeated configurations in the current population

Pop: Total number of configurations of the population.

\section{Acknowledgments}

This work was supported by CAPES, CNPq, and Fundação Araucaria.

\section{References}

[1] P. Paterni, S. Vitet, M. Bena, and A. Yokoyama, "Optimal location of phase shifters in the french network by genetic algorithm," IEEE Transactions on Power Systems, vol. 14, no. 1, pp. 37-42, 1999.

[2] G. N. Taranto, L. M. V. G. Pinto, and M. Veiga Ferraz Pereira, "Representation of FACTS devices in power system economic dispatch," IEEE Transactions on Power Systems, vol. 7, no. 2, pp. 572-576, 1992.

[3] S. Gerbex, R. Cherkaoui, and A. J. Germond, "Optimal location of multi-type FACTS devices in a power system by means of genetic algorithms," IEEE Transactions on Power Systems, vol. 16, no. 3, pp. 537-544, 2001.

[4] A. H. Escobar, R. A. Gallego, and R. Romero, "Multistage and coordinated planning of the expansion of transmission systems," IEEE Transactions on Power Systems, vol. 19, no. 2, pp. 735-744, 2004. 
[5] R. Fang and D. J. Hill, "A new strategy for transmission expansion in competitive electricity markets," IEEE Transactions on Power Systems, vol. 18, no. 1, pp. 374-380, 2003.

[6] A. Seifu, S. J. Salon, and G. F. List, "Optimization of transmission line planning including security constraints," IEEE Transactions on Power Systems, vol. 4, no. 4, pp. 1507-1513, 1989.

[7] S. Binato, M. V. F. Pereira, and S. Granville, "A new Benders decomposition approach to solve power transmission network design problems," IEEE Transactions on Power Systems, vol. 16, no. 2, pp. 235-240, 2001.

[8] R. A. Gallego, A. Monticelli, and R. Romero, "Transmision system expansion planning by an extended genetic algorithm," IEE Proceedings Generation, Transmission and Distribution, vol. 145, no. 3, pp. 329-335, 1998.

[9] E. L. D. Silva, H. A. Gil, and J. M. Areiza, “Transmission network expansion planning under an improved genetic algorithm," IEEE Transactions on Power Systems, vol. 15, no. 3, pp. 1168-1175, 2000.

[10] P. C. Chu and J. E. Beasley, "A genetic algorithm for the generalised assignment problem," Computers and Operations Research, vol. 24, no. 1, pp. 17-23, 1997.

[11] R. Villasana, L. L. Garver, and S. J. Salon, "Transmission network planning using linear programming," IEEE transactions on power apparatus and systems, vol. 104, no. 2, pp. 349-356, 1985.

[12] GARVER LL, "Transmission network estimation using linear programming," IEEE Trans Power App Syst, vol. 89, no. 7, pp. 1688-1697, 1970.

[13] S. Haffner, A. Monticelli, A. Garcia, and R. Romero, "Specialised branch-and-bound algorithm for transmission network expansion planning," IEE Proceedings Generation, Transmission and Distribution, vol. 148, no. 5, pp. 482-488, 2001.

[14] G. Latorre, R. D. Cruz, J. M. Areiza, and A. Villegas, "Classification of publications and models on transmission expansion planning," IEEE Transactions on Power Systems, vol. 18, no. 2, pp. 938-946, 2003.

[15] E. L. da Silva, J. M. A. Ortiz, G. C. de Oliveira, and S. Binato, "Transmission network expansion planning under a Tabu Search approach," IEEE Transactions on Power Systems, vol. 16, no. 1, pp. 62-68, 2001.

[16] H. Faria Jr, S. Binato, M. G. C. Resende, and D. M. Falcão, "Power transmission network design by greedy randomized adaptive path relinking," IEEE Transactions on Power Systems, vol. 20, no. 1, pp. 43-49, 2005. 

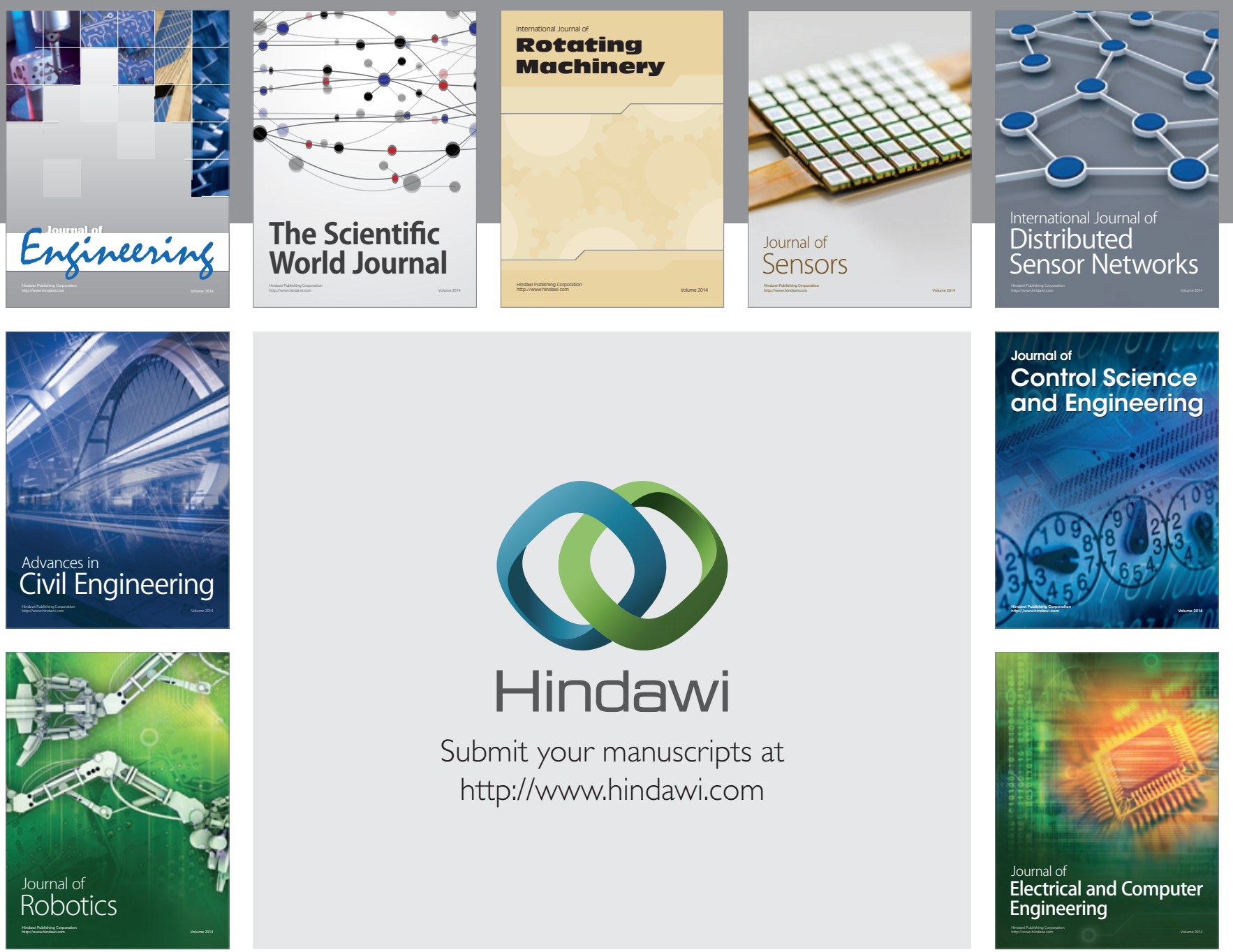

Submit your manuscripts at

http://www.hindawi.com
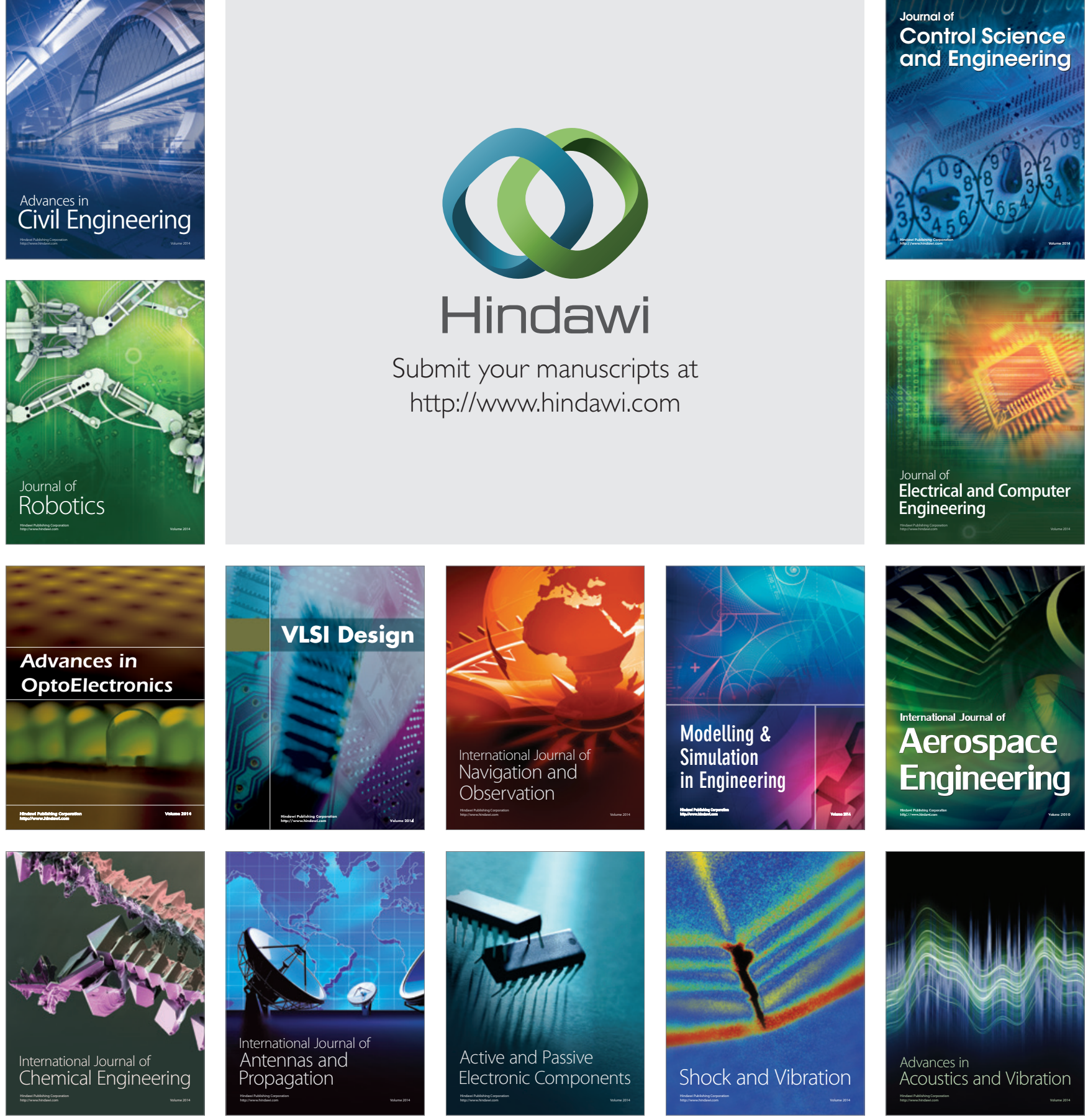